1

2

3

4

5

6

7

8

9

10

11

12

13

14

15

16

17

18

19

20

21

22

23

24

25 Correspondence:

26

27

28

29

30

31

32

33 2366, USA

Funding Source:

Professor

PO Box 162367

\section{Probabilistic Genotyping of Single Cell Replicates from Complex DNA Mixtures Recovers Higher Contributor LRs than Standard Analysis}

\author{
Kaitlin Huffman ${ }^{\mathrm{a}}$, Erin Hanson ${ }^{\mathrm{a}, \mathrm{b}, \mathrm{c}}$, Jack Ballantyne ${ }^{\mathrm{a}, \mathrm{b}, \mathrm{c}, *}$
}

${ }^{a}$ Graduate Program in Chemistry, Department of Chemistry, University of Central Florida, PO Box 162366, Orlando, FL 32816-2366, USA

${ }^{\mathrm{b}}$ National Center for Forensic Science, PO Box 162367, Orlando, FL 32816-2367, USA

${ }^{\mathrm{c}}$ Department of Chemistry, University of Central Florida, PO Box 162366, Orlando, FL 32816-

Declaration of interest:

No competing interests to disclose.

The authors would like to thank the State of Florida for initial seed funding for this project. The funders had no role in study design; in the collection, analysis, and interpretation of data; in the writing of the report; and in the decision to submit the article for publication.

Jack Ballantyne, $\mathrm{PhD}$

Department of Chemistry

University of Central Florida

Orlando, FL 32816-2367, USA

Email: Jack.Ballantyne@ucf.edu 


\section{Abstract}

DNA mixtures are a common source of crime scene evidence and are often one of the more difficult sources of biological evidence to interpret. With the implementation of

37 probabilistic genotyping (PG), mixture analysis has been revolutionized allowing previously unresolvable mixed profiles to be analyzed and probative genotype information from contributors to be recovered. However, due to allele overlap, artifacts, or low-level minor contributors, genotype information loss inevitably occurs. In order to reduce the potential loss of

41 significant DNA information from donors in complex mixtures, an alternative approach is to physically separate individual cells from mixtures prior to performing DNA typing thus obtaining single source profiles from contributors. In the present work, a simplified micromanipulation technique combined with enhanced single-cell DNA typing was used to collect one or few cells, referred to as direct single-cell subsampling (DSCS). Using this approach, single and 2-cell subsamples were collected from 2-6 person mixtures. Single-cell subsamples resulted in single source DNA profiles while the 2-cell subsamples returned either single source DNA profiles or new mini-mixtures that are less complex than the original mixture due to the presence of fewer contributors. PG $\left(\mathrm{STRmix}^{\mathrm{TM}}\right)$ was implemented, after appropriate validation, to analyze the original bulk mixtures, single source cell subsamples, and the 2-cell mini mixture

51 subsamples from the original 2-6-person mixtures. PG further allowed replicate analysis to be 52 employed which, in many instances, resulted in a significant gain of genotype information such

53 that the returned donor likelihood ratios (LRs) were comparable to that seen in their single 54 source reference profiles (i.e., the reciprocal of their random match probabilities). In every 55 mixture, the DSCS approach gave improved results for each donor compared to standard bulk 
mixture analysis. With the 5- and 6- person complex mixtures, DSCS recovered highly probative

57 LRs $\left(\geq 10^{20}\right)$ from donors that had returned non-probative LRs $\left(<10^{3}\right)$ by standard methods.

Abbreviations: DSCS (direct single cell subsampling); POI (person-of-interest); PG (probabilistic genotyping); NOC (number of contributors)

61

\section{Introduction}

DNA mixtures are a commonly encountered source of evidence found at crime scenes and are one of the more difficult sources of biological evidence to interpret due to their inherent complexity [1] as multiple genotype combinations can often explain the STR mixture data. This is particularly troublesome since much DNA evidence is comprised of a mixture originating from multiple contributors including the victim (s) and/or perpetrator(s) to the crime or to others who have no direct relationship to the incident in question [2-4]. With the implementation of probabilistic genotyping (PG), mixture deconvolution analysis has been revolutionized as PG is less subjective, more consistent, uses more DNA profile information, and provides greater statistical power resulting in the analysis of mixtures that were previously unresolvable $[5,6]$. contributors to a mixture is not always achieved [5] due to a loss of genotype information caused

74 by overlapping alleles, artifacts such as stutter, or very low-level minor contributors [7-10].

75 Furthermore, if the number of contributors (NOC) to a mixture is underestimated, information

76 about some of the donors cannot be inferred [3]. Other factors such as the relatedness of the

77 donors [11] or $\geq 4$ donors contributing to the mixture, can lead to even more complexity in allele

78 overlap resulting in further uncertainty of NOC. Even if the correct NOC is identified, not all 
79 donors will result in a probative LR value. Moreover, if a reference profile from a person of

80 interest (POI) is not available, analysis is severely limited as PG analysis alone does not truly

81 deconvolute mixtures into their single source genotypes in most instances, but rather provides the

82 likelihood (in the form of a likelihood ratio) that the DNA results indicate a specific genotype

83 could have contributed to the mixture at hand. Without a reference genotype to condition the LR

84 on, or an appropriate database to search against, actionable probative information cannot always

85 be obtained. This is particularly true of complex mixtures which can be defined as samples

86 containing comingled DNA from $\geq 2$ individuals where stochastic effects or allele sharing leads

87 to uncertainty in determining each of the contributors' DNA genotypes [12]. An exception to

88 this deconvolution issue would be the analysis of a simple 2-person mixture with clear

89 distinction of the major and minor contributor in which there were no significant stochastic and

$90 \quad$ low-template effects [13].

91 A potential solution to overcoming the issue of genotype information loss is to separate

92 individual cells or a small subset of cells from the original 'bulk' mixture prior to analysis

$93 \quad[10,14-21]$. Doing so may result in single source DNA profiles for all contributors as well as

94 new 'mini mixtures' with less complexity compared to the original bulk mixture due to a

95 decrease in the NOC [10]. In this study, this concept was applied to complex mixture analysis

96 using the previously reported direct single cell subsampling (DSCS) method, [10] but now,

97 analyzed with PG methods validated for enhanced DNA-STR typing of single (or few) cells.

\section{Methods}

\subsection{Sample collection}


101 Buccal swabs were collected from volunteers by swabbing the inside of the mouth and cheek

102 with a sterile cotton swab according to procedures approved by the University's Institutional

103 Review Board.

104

105

2.2. Slide creation

2.2.1. Gel-Film slide creation

107 Gel Film microscope slides were created by attaching Gel-Pak® Gel-Film ${ }^{\circledR}(\mathrm{WF}$, x8 retention

108 level) (Hayward, CA, USA) to clean glass microscope slides by the Gel-Film adhesive backing.

109 The clear protective covering was removed when creating mixture slides.

\subsubsection{Mixture slide creation}

112 Freshly collected or dried/frozen buccal swabs from individual donors were agitated in $300 \mu \mathrm{L}$ of

$113 \mathrm{TE}^{-4}$ buffer. The resulting solutions were centrifuged at $300 \mathrm{RCF}$ to create an epithelial cell

114 pellet. The supernatants were discarded, and $300 \mu \mathrm{L}$ of $\mathrm{TE}^{-4}$ buffer was added to resuspend each

115 cell pellet. The Countess ${ }^{\mathrm{TM}}$ II FL (ThermoFisher Scientific) automated cell counter was then

116 used according to manufacturer recommendations to determine the cell concentration of each of

117 these cell suspensions. Equals concentrations of each donor cell suspension were mixed to

118 create the desired mixtures (ie 2-, 3-, 4-, 5-, or 6-person). Mixture cell suspensions were stored

119 frozen. Once the desired mixture was created, $60 \mu \mathrm{L}$ of the mixture solution was pipetted onto

120 the Gel-Film ${ }^{\circledR}$ microscope slide and spread out using a sterile swab. The slide was then stained

121 with Trypan Blue for 1-2 min and gently rinsed with nuclease-free water. Slides were air-dried

122 overnight. 
$124 \quad 2.2 .3 .3 \mathrm{M}^{\mathrm{TM}}$ adhesive slide creation

125 Prior to cell collection, a 3M $\mathrm{M}^{\mathrm{TM}}$ (St. Paul, MN, USA) adhesive slide reservoir was made.

126 Double-sided tape was used to attach the $3 \mathrm{M}^{\mathrm{TM}}$ adhesive to a clean glass microscope slide, and

127 the adhesive backing was removed. The slide was then stored in a desiccator until needed

$128[22,23]$.

\subsection{Cell recovery}

131 Cells were visualized (from the gel-film mixture slide previously created in section 2.2.2) using a

132 Leica M205C stereomicroscope (190-240X magnification) and collected directly into sterile

$133 \quad 0.2 \mathrm{~mL}$ PCR flat-cap tubes containing Prep-n-Go ${ }^{\mathrm{TM}}$ Lysis buffer (ThermoFisher Scientific,

134 Carlsbad, CA, USA). This was done via a sterile tungsten needle and water-soluble $3 \mathrm{M}^{\mathrm{TM}}$

135 adhesive. The tungsten needle was used to obtain a small ball of $3 \mathrm{M}^{\mathrm{TM}}$ adhesive (from the

136 adhesive slide reservoir created in section 2.2.3) which was then used to adhere selected

137 visualized cells from the mixture slides [22,23]. Still under the microscope, the adhesive-tipped

138 tungsten needle containing adhered cells was inserted into an amplification tube $(0.2 \mathrm{~mL})$

139 containing $1 \mu \mathrm{L}$ lysis buffer until the $3 \mathrm{M}^{\mathrm{TM}}$ adhesive was observed to solubilize. Twenty 1 - and

140 2- cell subsamples were first collected and analyzed for every mixture. If the expected minimum

141 number of contributors (as determined from bulk analysis), or if probative log (LR) values were

142 not obtained for all donors, additional $\mathrm{s}=10 \times 1$ and 2 cell subsamples were collected until

143 probative results were achieved. Twenty 1 and 2-cell samples were collected for the 1:1 and

144 1:1:1 mixtures. Thirty 1 and 2-cell subsamples were collected for the 1:1:1:1 and 1:1:1:1:1:1

145 mixtures and forty 1 and 2-cell subsamples were collected for the 1:1:1:1:1 mixture. 


\subsection{Direct lysis/ autosomal short tandem repeat (STR) amplification of cells}

148 Cells were collected directly into $1 \mu \mathrm{L}$ Prep-n-Go ${ }^{\mathrm{TM}}$ lysis buffer as described in section 2.3.

149 Samples were then incubated at $90^{\circ} \mathrm{C} \rightarrow 20 \mathrm{mins} ; 25^{\circ} \mathrm{C} \rightarrow 15 \mathrm{mins}$. After lysis of cells, the

150 samples were amplified using the GlobalFiler ${ }^{\mathrm{TM}}$ Express kit (ThermoFisher Scientific, Carlsbad,

151 CA, USA) with a reduced reaction volume of $5 \mu \mathrm{L}$ and increased cycle number (32 cycles). The

152 GlobalFiler ${ }^{\mathrm{TM}}$ Express reaction mix was prepared consisting of $2 \mu \mathrm{L}$ PCR mix and $2 \mu \mathrm{L}$ primer

153 mix added to the $0.2 \mathrm{~mL}$ PCR flat-cap tubes. Samples were amplified using a protocol of

$15495^{\circ} \mathrm{C} \rightarrow 1 \mathrm{~min} ; 32$ cycles: $94^{\circ} \mathrm{C} \rightarrow 3 \mathrm{sec}, 60^{\circ} \mathrm{C} \rightarrow 30 \mathrm{sec} ; 60^{\circ} \mathrm{C} \rightarrow 8$ mins; $4^{\circ} \mathrm{C} \rightarrow$ hold. Positive (1

$155 \mu \mathrm{L} 31.25 \mathrm{pg} 007)$ and negative amplification controls (0-cell samples and amplification blanks)

156 were included in each amplification batch.

\subsection{Donor reference samples and bulk mixtures}

\subsubsection{DNA isolation and quantitation}

DNA was extracted from reference buccal swabs and $60 \mu \mathrm{L}$ of each mixture cell suspension using the QIAamp DNA Investigator kit (QIAGEN, Germantown, MD, USA) according to the manufacturer's manual extraction procedure. An extraction blank was included in each extraction set. Quantifiler ${ }^{\circledR}$ Duo DNA Quantification kit (ThermoFisher Scientific) was used to quantify extracts according to the manufacturer's recommended protocols using the Applied Biosystems’ 7500 real-time PCR instrument (ThermoFisher Scientific).

\subsubsection{Autosomal STR amplification (reference samples and bulk mixtures)}


170 The amplification protocol was: $95^{\circ} \mathrm{C} \rightarrow 1 \mathrm{~min} ; 29$ cycles: $94^{\circ} \mathrm{C} \rightarrow 10 \mathrm{sec}, 59^{\circ} \mathrm{C} \rightarrow 90 \mathrm{sec} ; 60^{\circ} \mathrm{C} \rightarrow$

$17110 \mathrm{mins} ; 4^{\circ} \mathrm{C} \rightarrow$ hold. A positive and negative amplification control was included in each

172 amplification.

173

\section{$174 \quad$ 2.6. PCR product detection}

175 One microliter of GlobalFiler ${ }^{\mathrm{TM}}$ or GlobalFiler $^{\mathrm{TM}}$ Express amplified product was added to $9.5 \mu \mathrm{L}$ $176 \mathrm{Hi}-\mathrm{Di}^{\mathrm{TM}}$ formamide (ThermoFisher Scientific) and $0.5 \mu \mathrm{L}$ GeneScan ${ }^{\mathrm{TM}} 600 \mathrm{LIZ} 囚$ size standard

177 (ThermoFisher Scientific). Samples were injected on an Applied Biosystems' 3500 Genetic

178 Analyzer using POP-4 ${ }^{\mathrm{TM}}$ polymer and Module G6 (15 s injection, $\left.1.2 \mathrm{kV}, 60^{\circ} \mathrm{C}\right)$. Samples were 179 analyzed using GeneMapper v1.6 software (ThermoFisher Scientific).

\subsection{Probabilistic Genotyping (PG)}

\subsubsection{Standard Bulk Mixture Probabilistic Genotyping}

183 Probabilistic Genotyping Software STRmix ${ }^{\mathrm{TM}}$ v2.8 was validated for use with standard bulk

184 mixtures and reference samples. Each bulk mixture was analyzed according to the known

number of donors. For example, for a 3-person mixture, the likelihood ratio was conditioned as

187 individuals. This approach was repeated for the remaining two donors. Probabilistic

188 Genotyping was also used to analyze all reference profiles as single source to determine the

189 random match probability. The sub-source $\log (\mathrm{LR})$ was reported. The five and six-person bulk

190 mixtures were run with the assistance of STRmix ${ }^{\mathrm{TM}}$ Technical and Scientific Support

191 (Environmental Science and Research) and a beta STRmix ${ }^{\mathrm{TM}}$ v2.9 for the 6-person mixture 
192 specifically. The FBI Caucasian database was used for all allele frequencies in all mixture

193 experiments.

\subsubsection{DSCS Probabilistic Genotyping} subsamples. Each 1-cell subsample was run as a single source sample and the $\log (\mathrm{LR})$ reported.

200 (from their training courses) was also utilized. Additional in-house analyst genotypes were

201 added to the database to monitor for contamination.

\subsubsection{DSCS Analytical Threshold}

The analytical threshold used when analyzing cell subsamples collected by DSCS was

207 lowest through) was used [24]. (Blue = 53 RFU; Green = 86 RFU; Yellow = 46 RFU; Red = 63

\subsubsection{DSCS Drop-in Rate}

The drop-in rate was calculated by analyzing 35 negative controls for the presence of

212 drop-in alleles. The equation used was the number of drop-in events/ (the number of loci scored

$213 \mathrm{x}$ the number of samples). The drop-in data did not fit a gamma distribution as limited drop-in

214 data was available. Therefore, a uniform distribution was utilized (graph not shown). A drop-in 
215 rate of 0.0164 and a drop-in cap of 30,000 RFUs was used to allow for any height allele to be

216 considered as possible drop-in. (This is an increase compared to the parameters used for the

217 standard bulk mixtures (bulk mixture: drop-in rate= $0.0001 \&$ cap= $100 \mathrm{RFU}$ )).

\subsubsection{DSCS STRmix ${ }^{T M}$ Stutter Parameters}

Stutter regression files were created for each locus and each type of stutter encountered

221 according to the STRmix ${ }^{\mathrm{TM}}$ implementation and validation guide. The STRmix ${ }^{\mathrm{TM}}$ stutter

222 parameters were then determined using the Model Maker function. Model Maker utilized $161 \mathrm{x}$

$223-1,-2,-3,-4$, or 5-cell subsamples to model the stutter distribution. The maximum allowed

224 stutter percentages were larger than those typically seen with standard analysis because of

225 elevated stutter that is characteristic of low template stochasticity. The DSCS stutter parameter

226 results are shown in Supplementary Table 1. For comparison, the stutter parameters used for the

227 standard bulk mixtures are provided in Supplementary Table 2.

\subsubsection{DSCS STRmix ${ }^{T M}$ Post Burn-in Accepts}

A single source 3-cell subsample was analyzed 5 times using 5,000, 50,000, and 500,000

231 post burn-in accepts. It was determined that 500,000 post burn-in accepts gave the most

232 consistent $\log (\mathrm{LR})$ results (graph not shown) for DSCS samples. Additional DSCS settings

233 (including, for comparison, those for standard bulk mixtures) are provided in Supplementary

234 Table 3.

235 
After PG analysis of each subsample, replicate analysis [25] was performed per donor for single source 1-cell samples, single source 2-cell samples, and 2-cell mini-mixtures. The top up

to six 1-cell subsamples that gave a $\log (\mathrm{LR}) \mathrm{s} \geq 1$ per donor were utilized for 1-cell replicate analysis. These 1-cell subsample replicates were run as single source ie. LR= Donor 1 vs.

241 unknown individual. Likewise, the top up to six single source 2-cell subsamples that gave log

242 (LR)s $\geq 1$ for a donor were utilized for 2-cell replicate analysis. Two-cell mini-mixture

243 replicates were conducted any time a $\log (\mathrm{LR}) \geq 1$ was achieved for a donor regardless of

244 identity of the second donor.

\subsection{Description of DSCS Method}

An infographic of the DSCS method for complex mixture analysis that uses an equimolar

248 4-person mock mixture for illustrative purposes is provided in Fig. 1. The standard bulk mixture

249 analysis, which begins with the extraction and de facto homogenization of DNA from a mixed

250 stain, results in an admixture of DNA from contributors where each contributor's genotype

251 cannot be distinguished (Fig. 1, left side). Direct single cell subsampling of the same mixture

252 allows for collection of multiple 1-2 cell subsamples. This allows for single source profiles of all

253 individuals (A, B, C, and D) from the mixture to be obtained by 1-cell subsamples as well as

254 some 2-cell subsamples. By increasing the number of cells collected in subsampling from 1 to 2 ,

255 the amount of input DNA doubles thus increasing the probability of achieving a full profile if

256 both cells originate from the same donor. However, some 2-cell subsamples still result in

257 admixed profiles (i.e. 2-cell mini-mixtures) which are less complex than the original bulk

258 mixture due to a decrease in the number of contributors (Fig. 1, right side). 
The direct single cell subsampling method produces a number of different subsample

260 types that are then analyzed with probabilistic genotyping (PG). Fig. 2 shows an infographic

261 summarizing the PG analysis decision tree developed in this study for the subsample types using

262 the same 4-person mock DNA mixture shown in Fig. 1. Prior to DSCS analysis, a bulk mixture is

263 analyzed using standard methods. With DSCS, a collection of single cell subsamples results in

264 single source profiles of donors to the mixture. These individual subsample DNA profiles from

265 single cells are then analyzed using PG techniques validated for use with direct PCR, reduced

266 reaction volumes and few cells. For this, each recovered subsample genotype is PG tested

267 individually against all potential donors comprising the mixture. Thus, for each subsample a

268 number of LRs, dependent upon the number of potential donors tested as a contributor, will be

269 calculated. Any subsample profile that gives a $\log (\mathrm{LR}) \geq 1$ for a specific donor is then deemed

270 suitable for PG replicate analysis for that specific donor, and the presence of at least one other

271 subsample with a positive $\log$ (LR) for the same donor is needed for PG replicate analysis. By

272 this process the genotyped subsamples from the mixture are essentially categorized according to

273 putative donor prior to conducting the replicate analysis. The same process is conducted for 2-

274 cell single source subsamples. For 2-cell mixture samples, i.e. 'mini-mixtures', replicate analysis

275 is conducted when multiple mixture samples give a $\log (\mathrm{LR}) \geq 1$ for a specific donor. For

276 simplicity, the subsequent use of the term DSCS in this report is taken to mean the PG validated

277 DSCS method.

278

279 3. Results

280

281

\subsection{STR kits/lysis conditions}


282

283

284

285

286

287

288

289

290

291

292

293

294

295

296

297

298

299

300

301

302

303

304

For DSCS, more discriminatory and sensitive commercial STR kits than previously reported [10] were tested using 1-, 2-, 3-, 4-, 5- and 10-cell subsamples. Based upon the quality of the profiles and the number of alleles recovered (Supplementary Fig. 1), two kits were found to perform particularly well, namely GlobalFiler ${ }^{\circledR}$ Express (GFE) and PowerPlex ${ }^{\circledR}$ Fusion 6C (PPF6C). GFE was chosen as the system of choice for PG validation in this study since it performed best for single buccal cells and was designed specifically for direct PCR, which is used in the DSCS process. However, PPF6C represents a suitable alternative sensitive STR system that can be used for single cell STR typing. Examples of GlobalFiler ${ }^{\circledR}$ Express 1 and 2 cell EPGs can be seen in Supplementary Figs. 2, 3, and 4.

\subsection{PG validation}

The PG software system STRmix ${ }^{\mathrm{TM}}$ was validated using GlobalFiler ${ }^{\circledR}$ (GF) for standard mixture analysis according to the recommendations of the software developers $[5,26]$. For use with DSCS, PG validation was conducted using the same methodology except with subsamples of discrete nucleated cells as input (1-5 cells, equivalent to approximately 6.6 pg-33 pg DNA) typed with GlobalFiler ${ }^{\circledR}$ Express (GFE) in contrast to standard applications which utilized DNA input ( 0.1-1.0 ng gDNA) arising from dilutions of bulk DNA extracts. The STRmix ${ }^{\mathrm{TM}}$ validation comprised two phases, namely the determination of empirically derived parameters used to formulate a model and subsequent testing of the sensitivity and specificity of the model to deconvolute known mixtures.

As an additional test of the accuracy of the PG parameters for 1-5 cells, a 3-cell single source sample was analyzed using probabilistic genotyping, and then, an artificially degraded profile (created by decreasing the peak heights of the high molecular weight alleles by $80 \%$ and 
the low molecular weight alleles by 5\%), an artificially inhibited profile (created by decreasing the peak heights of D22S1045, D21S11, D13S317, and D2S1338 loci by 40\%), and a profile with artificial drop-in (created by adding a drop-in 8 allele at D5S818 with 15,000 RFUs) were created and analyzed. A single dye channel from the degraded, inhibited, and drop-in profiles is shown in Supplementary Fig. 5. In all 3 artificial profiles, PG reported the same log (LR) as the original sample (Supplemetary Table 4).

\subsection{Testing the limits of standard PG analysis with complex mixtures}

With the PG systems validated for standard DNA mixture analysis (i.e. 'bulk' analysis rather than subsampling by DSCS) we tested the limits of a commonly used PG system in

315 extracting probative information from standard mixtures. To that end we selected random 2, 3, and 4 person DNA mixtures (50 from each mixture class) from in-house prepared DNA mixtures

317 and the PROVEDIt database [27,28] and calculated the $\log (\mathrm{LR}) \mathrm{s}$ for each known donor using

318 STRmix ${ }^{\mathrm{TM}}$. The mixtures comprised a variety of donors and mixture weight ratios.

319 Quantitatively, in these 2-,3-, and 4-person mixtures, only 86\%, 74\%, and $32 \%$ of each respective mixture type gave very strong support $(\log (L R) \geq 6)$ for each contributor (Table 1$)$.

Note that a similar number of 5- and 6-person mixtures, albeit 5-person mixtures are

322 available on the PROVEDIt database, could not return LRs in this study due to computational

323 speed and memory limitations. Indeed, we were able, with the help of a supercomputer and

324 STRmix ${ }^{\mathrm{TM}}$ Technical and Scientific Support (Environmental Science and Research), to perform 325 standard PG analysis on an in- house generated 5- and 6-person mixture. In both mixtures, the 326 LRs from three of the known contributors failed to reach the $\log (\mathrm{LR})=6$ threshold. 
These results indicate that the DSCS approach, if successful, could be of benefit in

recovering more highly probative genotype information from each of the contributors to complex mixtures.

\subsection{Sensitivity and Specificity of DSCS}

Since DSCS is dependent upon analysis of low template samples, a high degree of dropaverage 18 alleles while 2-cell single source subsamples recovered 26. This high degree of dropout can occasionally result in profiles with high allele counts but low LRs principally due to single allele drop-outs from heterozygous loci. Since the simultaneous deconvolution of

337 replicates within a single analysis reduces the impact of stochastic effects [25], the available replicate analysis capability with PG systems was further investigated for DSCS applicability.

The sensitivity of PG for single cell genotyping by DSCS was assessed. Sensitivity is defined in PG analysis as the proportion of true contributors that return positive LR values (i.e.

341 lends support for the inclusionary proposition $\mathrm{H}_{1}$ or $\mathrm{H}_{\mathrm{p}}$ ). The LR threshold for most sensitivity

342 studies has traditionally been set at 1 (i.e. $\log (\mathrm{LR})=0)$ and thus any sample that returns a $\log$

$343(\mathrm{LR})>0$ would be regarded as a positive for the sensitivity analysis. In this work, since the aim

344 is to better recover highly probative donor genotype information from complex mixtures, we also 345 assessed the ability of the DSCS procedure to achieve a minimum log (LR) of 6 for individual 346 cell subsamples from each mixture contributor. We chose this latter LR threshold since it 347 excludes the possibility of obtaining false positive results from (unrelated) non-contributors, and 348 represents the upper 'evidentiary significance' threshold beyond which the international forensic community has decided no additional verbal expressions beyond providing "extremely strong 
support" for the inclusionary proposition are applied [29,30]. Equimolar 2-6 person mixtures were prepared from buccal cells as described (Methods Section 2.2.2) and a total of 291 cell subsamples $(110 \times 1$ cell subsamples, $81 \times$ 2-cell single source subsamples, $100 \times 2$-cell mini mixtures) were recovered by DSCS and subjected to PG analysis with STRmix. Table 3 shows $>0$ and the $\log (\mathrm{LR}) \geq 6$ targeted in this work. majority of 2-cell single source subsamples (84\%), 1-cell subsamples (68\%), and 2-cell mini mixture subsamples (60\%). While some of the subsamples were not of a high enough quality to reach the 'very strong support' threshold for the inclusionary hypothesis $(\log (\mathrm{LR}) \geq 6)$, the majority (60\%) of 2-cell single source subsamples, $26 \%$ of 1-cell subsamples, and 29\% of 2-cell mini mixture subsamples did so. Significantly, some 1 and 2-cell single source subsamples

362 returned $\log (\mathrm{LR}) \mathrm{s} \geq 21$ (3\% and $19 \%$ respectively), which is close to the inverse of the match 363 probability for the donors. The latter effectively represents the upper bound of the achievable LR [31] and therefore also the maximum information recoverable from the subsample. sensitivity of DSCS analysis. Cell subsamples are low template DNA samples and replicate

367 analysis of low template samples has been shown to not only reduce characteristic confounding 368 stochastic effects [32] but to be able to obtain higher LRs from multiple low template replicates 369 than from a single bulk sample [31]. Sensitivity was significantly improved (i.e. LRs increased) 370 when replicate analysis was performed on the DSCS subsamples. Table 3 shows the results when 371 the same samples as above were subjected to replicate analysis for which, on average, 4 372 subsamples $( \pm 1)$ exhibiting the highest returned LRs were used. Replicate analysis resulted in 
$100 \%$ sensitivity for all subsample types when the threshold was set at $\log (\mathrm{LR})>0$. In almost all instances, returned LRs provided 'very strong support' (i.e. $\log (\mathrm{LR}) \geq 6$ ) for the inclusionary hypothesis (94\% for 1-cell replicates, $93 \%$ for 2 -cell single source replicates, and $86 \%$ of 2 -cell mini mixture replicates). Significant proportions of 1- and 2-cell single source replicates resulted in $\log (\mathrm{LR}) \mathrm{s} \geq 21$ (41\% for 1 -cell replicates, $79 \%$ for 2 -cell replicates) (data not shown). Specificity is the proportion of true non-contributors that return $L R<1$ values (i.e. $\log$ $(\mathrm{LR})<0)$ and thus supports the exclusionary proposition $\left(\mathrm{H}_{2}\right.$ or $\left.\mathrm{H}_{\mathrm{d}}\right)$. The specificity of DSCS analysis was checked for each single source 1-cell and 2-cell subsamples used in the sensitivity analysis. This was done by substituting 1000 random known non-contributor DNA profiles instead of the known contributors (i.e. substituting the non-contributor for the known in the inclusionary proposition (i.e. $\mathrm{H}_{1}$ or $\mathrm{H}_{\mathrm{p}}$ )) and calculating the $\mathrm{LR}$ for each. The results are shown in Figure 3 in which the log (LR) values for known donors are plotted for each subsample with respect to the number of alleles detected. Thus, each single source 1 and 2 cell subsample (blue and yellow respectively) will return a single LR for the known contributor and 1000 LRs for the non-contributors (orange). Likelihood ratios of 0 were plotted as a $\log (\mathrm{LR})=-50$. As before, the $\log (\mathrm{LR})$ achieved from known donors to single source subsamples increased as allele recovery increased while the number of non-contributor false positives with an LR $>1$ also decreased as allele count increased. As can be seen from Fig. 3, non-contributors never exceeded the $10^{6} \mathrm{LR}$ threshold (dashed line) although several cells with less than 10 alleles returned LRs $>1$. The majority of false positives had a log (LR) between 1 to 3 indicating 'uninformative' or 'limited support' [29,30] but one non-contributor subsample did result in a log (LR) of 5. There was good separation between the $\log (\mathrm{LR}) \mathrm{s}$ obtained from known and noncontributors when the detected number of alleles from the cell(s) exceeded 15. Similarly, the log 
(LR)s achieved with known donors and known non-contributors to 2-cell mini mixture

subsamples were plotted according to the number of non-shared alleles. No false positives were obtained at or above a threshold of $\log (\mathrm{LR})=6$ (Supplementary Fig. 6).

Improved specificity and sensitivity of DSCS was obtained by replicate analysis (Fig. 4). analysis increases with replicate analysis and can result in the recovery of most (sometimes all) donor genotype information.

\subsection{DSCS applied to complex mixtures}

414 to complex mixtures was investigated using equimolar, buccal 2-6 person admixtures. The

415 replicate $\log (\mathrm{LR}) \mathrm{s}$ from DSCS analysis was determined as previously described (section 2.7.3)

416 for each contributor by using up to $6 \log (\mathrm{LR}) \geq 1$ subsamples (i.e. the highest $\operatorname{six} \log (\mathrm{LR}) \mathrm{s}$ or, if

417 less than 6 replicates with a $\log (\mathrm{LR}) \geq 1$ are obtained, all replicates are used). Supplementary

418 Figs. 8 and 9 shows vertical bar graphs of the replicate analysis log (LR) results for each 
contributor per mixture (heights of the bars) as well as the individual subsample log (LR) results that were used as replicates (dots within contributor bars). Across all mixtures shown the average number of subsample replicates used in the replicate analysis per donor per mixture was 4, with a median of 3 .

The DSCS replicate log (LR)s were compared to those from the ground truth single source samples (i.e. $\log (1 / \mathrm{RMP}$ from reference samples)) and that obtained from standard bulk mixture DNA analysis using STRmix ${ }^{\mathrm{TM}}$ (Figs. 5 and 6). DSCS recovered more genotype information from every donor in every mixture compared to standard PG mixture analysis, with the LR gain being noticeably more significant in the 5 and 6 person mixtures. One of the donors to the 4-person mixture (FM20), three donors to the 5-person mixture (S3, S5 and FM20), and three donors to the 6-person mixture (S8, CM31 and SA10) failed to return a $\log (\mathrm{LR}) \geq 6$ with standard analysis whereas DSCS returned highly probative log (LR)s from the same donors (range 8-27). In some donors, recovery of their full intrinsic genotype information (i.e. log $\left.(\mathrm{LR})_{\mathrm{DSCS}}=\log (1 / \mathrm{RMP})\right)$ was achieved. An additional 6-person mixture was analyzed as can be seen in Supplementary Fig. 10. and highly probative log (LR)s were recovered by DSCS from every donor (range 13-24). No standard bulk mixture analysis results were available for comparison with this mixture due to computational limitations.

\section{Discussion}

We reasoned that the use of continuous probabilistic genotyping (PG) methods and models, which use empirically derived variance parameters and can perform replicate analysis, could be productively employed in single cell analysis to provide accurate and reliable genotype resolution and LR (i.e. evidential weight) estimates. In this proof-of-concept (PoC) work, we 
442 have shown that direct single cell subsampling (DSCS) of complex DNA mixtures, combined

443 with high sensitivity DNA typing followed by PG interpretation, can recover more genotype

444 information (as demonstrated by increased LRs) from each of the contributors than that obtained

445 by standard methods. We define complex mixtures in this study as samples that contain DNA

446 from two or more contributors in which stochastic effects or allele sharing cause uncertainty in

447 determining contributor genotypes [12]. The information gain was achieved because single-cell

448 subsamples from the mixtures resulted in deconvoluted single-source DNA profiles while 2-cell

449 subsamples returned either single-source DNA profiles or new mini-mixtures that are less

450 complex than the original mixture due to the presence of fewer contributors. A suitably validated

451 PG software system, STRmix ${ }^{\mathrm{TM}}$, was implemented to analyze the original bulk mixtures, single-

452 source cell subsamples, and the 2-cell mini mixture subsamples obtained from the 2-6 person

453 complex mixtures studied. In every mixture, the DSCS approach gave improved results for each

454 donor compared to standard bulk mixture analysis. With 5- and 6- person complex mixtures,

455 DSCS recovered highly probative LRs $\left(>10^{20}\right)$ from donors that had returned non-probative LRs

$456\left(<10^{3}\right)$ by standard methods. In many such instances, the returned donor likelihood ratios (LRs)

457 were comparable to that seen in their single-source reference profiles. We employed a different

458 single cell-validated PG system, EuroForMix (v3.1.0, Quantitative LR MLE based) [9,33] to

459 analyze the mixtures. The two different PG software systems returned remarkably similar

460 individual contributor LR values with the DSCS samples with no significant discordant results

461 (data not shown). This represented an additional diagnostic check [34] on the accuracy and

462 reliability of the DSCS method's performance with the sample set studied. The DSCS method

463 with replicates represents a better and more objective approach to donor assignment and 
464 interpretation of single cell subsamples than previous methods [10] which principally used manual consensus profiling from replicates to recover genotype information. Maximum information gain was achieved by taking advantage of replicate contributor

467 subsamples that are intentionally obtained by the DSCS process and the subsequent use of the 468 replicate analysis capability of the PG systems. Consensus DNA profiles obtained from low 469 template analysis of bulk extracted samples, given enough replicates, can reconstitute much of 470 the genotype information that would otherwise be lost due to low template DNA stochastic 471 processes [32,35]. Here we employed replicate analysis to do the same thing for DSCS single 472 cell subsamples using the replicate analysis capabilities of the PG systems. The way replicates work here is that cell subsamples are genotyped and clustered/classified essentially according to

474 genotype, by performing PG analysis on all individual subsamples that yield STR profiles and 475 determining which, if any, return an LR $\geq 1$ for any of the potential POIs (Fig. 2). In order to 476 test the PG system's replicate accuracy, whereby a subsample might be misclassified for replicate 477 use, simulations were carried out in which a single subsample (with LR <1) collected from the 478 same bulk mixture but from a different donor (i.e. incorrect donor for the replicates being 479 analyzed) was added to a replicate subset originating from a particular donor and replicate analysis performed: this was repeated by substituting other single subsamples with an LR $<1$

481 (data not shown). When including misclassified samples for single source replicate analysis, if a 482 poor-quality profile was used ( $\sim 4$ alleles from a possible 42$)$, relatively no change was seen in 483 the $\log (\mathrm{LR})$ achieved. As profile quality of the misclassified subsamples increased, positive log 484 (LR)s of the replicates were no longer obtained (LRs of 0 or strongly negative). When including 485 misclassified subsamples for mini-mixture replicate analysis, sometimes, a positive result for the 486 true donors was still seen, but it did not increase the LRs obtained without the use of replicate 
487

488

489

490

491

492

493

494

495

496

497

498

499

500

501

502

503

504

505

506

507

508

509

analysis. Thus, it appears that the classification of subsamples with an $\log (\mathrm{LR}) \geq 1$ by PG into separate putative contributor clusters that are then suitable for PG replicate analysis is a valid approach at this time. Other subsample classification systems based upon genetic distance considerations are currently under investigation.

In a single mixture, the DSCS procedure produces up to six different subsample types with associated LRs from separate putative contributors. These subsample types include 1- and 2-cell subsamples, 2-cell mini-mixture subsamples, 1- and 2-cell replicates, and mini-mixture replicates. After replicate analysis, the maximum replicate likelihood ratio obtained is reported. While the results are encouraging, more work is required before the DSCS method would be suitable for casework applications. The data set is limited, with 5 mixtures (2-6 persons) being analyzed in detail by DSCS, although approximately 300 separate STR genotyping reactions were performed on the mixture subsamples (40-80 per complex mixture) and several hundred more for the PG validation studies. Thus, a wider range of complex mixtures with differing mixture contributor ratios as well as environmentally compromised cells and different cell types (including vaginal epithelia, white blood cells and cells from touch DNA deposits) should be investigated to determine whether a similar improvement in contributor genotype information recovery compared to standard bulk mixture analysis is achieved.

It should be emphasized that DSCS, even if subsequent studies confirm its accuracy and value with bona fide forensic samples, is not intended to replace current standard methods of mixture analysis. Instead, it is intended to be used for complex mixtures only after a standard ('bulk') mixture analysis is performed, which results in a determination of the minimum number of contributors and the computed LRs for the POIs. Only then, a determination would be made as to whether DSCS would be worthwhile pursuing in an attempt to retrieve more probative 
510 information from the sample. Of course, an appropriate sampling strategy would have to be

511 developed, validated and employed to permit subsequent DSCS analysis after standard analysis

512 (without compromising the latter). For example, loosely adhering deposited cells on the

513 underlying substrate could be transferred directly [17] or indirectly (via a cotton swab) onto the

514 inert silicone Gel-film ${ }^{\mathrm{TM}}$ before or after standard bulk mixture analysis. DSCS's main use would

515 be retrospective for those more complex mixture cases in which one (or more) POI contributor(s)

516 has returned an LR that did not provide "extremely strong support" for the inclusionary

517 hypothesis (e.g., did not reach the internationally recognized threshold of a $\log (\mathrm{LR}) \geq 6$, beyond

518 which the verbal conclusion does not change $[29,30])$ or in cases where at least one POI was not

519 present in a high enough relative proportion for standard DNA mixture analysis methods to

520 retrieve an STR profile. Also, the method could prove to be particularly useful for those

521 complex mixture cases in which multiple and/or alternative first-degree relatives are the POI(s).

522 Since the DSCS method uses morphologically distinguishable features to recognize cells

523 for recovery, a potential concern would be whether using the method in real forensic

524 physiological dried stains might therefore fail to pick up all DNA contributors to the mixture due

525 to the presence of cell free DNA (cfDNA) [36] which would be missed if only cells were

526 collected from the sample. The authors do not believe that the presence of cfDNA per se

527 invalidates the proposed DSCS approach. Even if there were cfDNA contributors to a mixture in

528 the absence of concomitant deposited cells, their presence would be detected by standard bulk

529 analysis (assuming the cfDNA was of sufficient quality and quantity for STR analysis, otherwise

530 its presence would be moot). So, in these type of mixtures DSCS would fail to detect the

531 cfDNA contributor(s) and improved genotype information recovery from that individual (or

532 individuals) by DSCS would not be possible. However, the ability to recover more genotype 
information would remain the same for any other contributors to the same mixture that did have cells present, including from those that may not have been detectable by standard methods due to a very low mixture ratio. Indeed, arguably there would now be a higher probability of recovering genotype information by DSCS from a minor sub-analytical contributor since the

537 mixture ratio of this minor donor in the cellular fraction would now be increased compared to 538 that in the bulk mixture (a proportion of which would comprise cfDNA from a non-cellular 539 donor).

The question arises as to whether the results obtained from DSCS would have limited

541 utility since they cannot be entered into searchable crime scene and criminal databases such as

542 CODIS. Currently, results of standard complex mixture analysis cannot be input into CODIS

543 either. This is because deconvolution into the constituent genotypes with these mixtures using

544 PG is imprecise in that for every locus there are usually several genotype combinations that

545 could feasibly (albeit with different probabilities) explain the results. An exception to this would

546 be where there is a clear and easily resolvable major donor, but unfortunately, even in these

547 cases, the POI may not be the major donor. Very often the most probable multi-locus genotype

548 inferred by the PG system is not exactly that of the POI. This uncertainty of the genotype

549 obtained by PG currently precludes the ability to input a particular source genotype into CODIS.

550 Notwithstanding the above, there are ongoing efforts to produce software to enable standard PG

551 mixture analysis results to search CODIS type databases in the future (e.g. STRmix's DBLR ${ }^{\mathrm{TM}}$

552 investigative tool). The latter envisions the possibility of a local search of the crime scene

553 mixture by interrogating individuals from the criminal database via a rapid calculation of LRs

554 from each of the individuals (or subsets thereof). However, with the DSCS method, single source 555 genotypes can be obtained and, arguably, there would actually be a better chance of being able to 
interrogate donors of complex mixtures against CODIS than the current method. This scenario however would have to be accompanied by extensive community validation of the low template methods employed by DSCS which admittedly would require extra work on the part of the

559 broader community. Many labs are, in reality, using low template methods currently due to the use of high sensitivity kits and the fact that PG deals routinely with the stochastic effects common to low template samples [37]. The path to CODIS or other regulated database acceptability may be long and narrow for DSCS approaches employing high sensitivity methods,

563 but it is not impossible, since there are published guidelines on how to validate them [38].

If a community developmental validation of DSCS with more casework relevant samples proved successful, DSCS using simplified micromanipulation as used here could readily be

566 implemented in a casework laboratory due to it only requiring basic equipment and materials and

567 its ease of use. Alternatively, the use of a semi-automated digital cell sorting method [18] could

568 be employed for those labs that prefer an instrument-versus microscope-based approach. The PG 569 software used in this study is perhaps already being used or validated in the laboratory.

In summary, the study establishes a method that could potentially overcome limitations to 571 the current DNA mixture analysis paradigm. The physical sampling with PG analysis of captured 572 cells permits the recovery of most (or all) of the contributor STR genotype information contained

573 within a complex mixture. Indeed, low level contributors that may not even be detectable by 574 standard methods could benefit from a subsampling method such as proposed here to retrieve the 575 otherwise totally lost information. Eventual implementation of such a DSCS approach would be 576 expected to increase the number of complex DNA mixture cases in which a person of interest is 577 definitively implicated or excluded as being a contributor to the biological trace. 


\section{Acknowledgments}

The authors would like to thank the anonymous donors who provided samples for this study and STRmix $^{\mathrm{TM}}$ Technical and Scientific Support for assistance answering technical questions related to the PG system as well as Catherine McGovern (Environmental Science and Research) for assistance in running the 5-person bulk mixture and the 6-person bulk mixture with STRmix ${ }^{\mathrm{TM}}$ v2.9.

\section{References}

[1] Y. Torres, I. Flores, V. Prieto, M. López-Soto, M.J. Farfán, A. Carracedo, P. Sanz, DNA mixtures in forensic casework: a 4-year retrospective study, Forensic Sci. Int. 134 (2003) 180-186. https://doi.org/10.1016/S0379-0738(03)00161-0.

[2] M.W. Perlin, M.M. Legler, C.E. Spencer, J.L. Smith, W.P. Allan, J.L. Belrose, B.W. Duceman, Validating TrueAllele ${ }^{\circledR}$ DNA Mixture Interpretation* $\uparrow$, , J. Forensic Sci. 56 (2011) 1430-1447. https://doi.org/10.1111/j.1556-4029.2011.01859.x.

[3] D.W. Bauer, N. Butt, J.M. Hornyak, M.W. Perlin, Validating TrueAllele ® Interpretation of DNA Mixtures Containing up to Ten Unknown Contributors, J. Forensic Sci. 65 (2020) 380-398. https://doi.org/10.1111/1556-4029.14204.

[4] J. Yang, D. Lin, C. Deng, Z. Li, Y. Pu, Y. Yu, K. Li, D. Li, P. Chen, F. Chen, The advances in DNA mixture interpretation, Forensic Sci. Int. 301 (2019) 101-106. https://doi.org/10.1016/j.forsciint.2019.05.024.

[5] T.R. Moretti, R.S. Just, S.C. Kehl, L.E. Willis, J.S. Buckleton, J.-A. Bright, D.A. Taylor, A.J. Onorato, Internal validation of STRmix ${ }^{\mathrm{TM}}$ for the interpretation of single source and mixed DNA profiles, Forensic Sci. Int. Genet. 29 (2017) 126-144. 
https://doi.org/10.1016/j.fsigen.2017.04.004.

[6] M.D. Coble, J.-A. Bright, Probabilistic genotyping software: An overview, Forensic Sci. Int. Genet. 38 (2019) 219-224. https://doi.org/10.1016/j.fsigen.2018.11.009.

[7] J.-A. Bright, D. Taylor, J.M. Curran, J.S. Buckleton, Developing allelic and stutter peak height models for a continuous method of DNA interpretation, Forensic Sci. Int. Genet. 7 (2013) 296-304. https://doi.org/10.1016/j.fsigen.2012.11.013.

[8] J. Ballantyne, E.K. Hanson, M.W. Perlin, DNA mixture genotyping by probabilistic computer interpretation of binomially-sampled laser captured cell populations: Combining quantitative data for greater identification information, Sci. Justice. 53 (2013) 103-114. https://doi.org/10.1016/j.scijus.2012.04.004.

[9] Ø. Bleka, C.C.G. Benschop, G. Storvik, P. Gill, A comparative study of qualitative and quantitative models used to interpret complex STR DNA profiles, Forensic Sci. Int. Genet. 25 (2016) 85-96. https://doi.org/10.1016/j.fsigen.2016.07.016.

[10] K. Huffman, E. Hanson, J. Ballantyne, Recovery of single source DNA profiles from mixtures by direct single cell subsampling and simplified micromanipulation, Sci. Justice. 61 (2021) 13-25. https://doi.org/10.1016/j.scijus.2020.10.005.

[11] M. Semaan, S. Abbas, I. Mansour, A Mixed DNA Profile Controversy, J. Forensic Res. 11 (2020). https://www.hilarispublisher.com/open-access/a-mixed-dna-profilecontroversy.pdf.

[12] J.M. Butler, H. Iyer, R. Press, M.K. Taylor, P.M. Vallone, S. Willis, DNA Mixture Interpretation: A NIST Scientific Foundation Review, (2021). https://doi.org/https://nvlpubs.nist.gov/nistpubs/ir/2021/NIST.IR.8351.

[13] P. Gill, C.H. Brenner, J.S. Buckleton, A. Carracedo, M. Krawczak, W.R. Mayr, N. 
Morling, M. Prinz, P.M. Schneider, B.S. Weir, DNA commission of the International Society of Forensic Genetics: Recommendations on the interpretation of mixtures, Forensic Sci. Int. 160 (2006) 90-101. https://doi.org/10.1016/j.forsciint.2006.04.009.

[14] P. Gill, A.J. Jeffreys, D.J. Werrett, Forensic application of DNA 'fingerprints,' Nature. 318 (1985) 577-579. https://doi.org/10.1038/318577a0.

[15] K. Anslinger, B. Bayer, Whose blood is it? Application of DEPArray ${ }^{\mathrm{TM}}$ technology for the identification of individual/s who contributed blood to a mixed stain, Int. J. Legal Med. 133 (2019) 419-426. https://doi.org/10.1007/s00414-018-1912-7.

[16] L. Dean, Y.J. Kwon, M.K. Philpott, C.E. Stanciu, S.J. Seashols-Williams, T. Dawson Cruz, J. Sturgill, C.J. Ehrhardt, Separation of uncompromised whole blood mixtures for single source STR profiling using fluorescently-labeled human leukocyte antigen (HLA) probes and fluorescence activated cell sorting (FACS), Forensic Sci. Int. Genet. 17 (2015) 8-16. https://doi.org/10.1016/j.fsigen.2015.03.003.

[17] K. Farash, E.K. Hanson, J. Ballantyne, Single source DNA profile recovery from single cells isolated from skin and fabric from touch DNA mixtures in mock physical assaults, Sci. Justice. 58 (2018) 191-199. https://doi.org/10.1016/j.scijus.2017.12.006.

[18] F. Fontana, C. Rapone, G. Bregola, R. Aversa, A. de Meo, G. Signorini, M. Sergio, A. Ferrarini, R. Lanzellotto, G. Medoro, G. Giorgini, N. Manaresi, A. Berti, Isolation and genetic analysis of pure cells from forensic biological mixtures: The precision of a digital approach, Forensic Sci. Int. Genet. 29 (2017) 225-241. https://doi.org/10.1016/j.fsigen.2017.04.023.

[19] N.A. Stokes, C.E. Stanciu, E.R. Brocato, C.J. Ehrhardt, S.A. Greenspoon, Simplification of complex DNA profiles using front end cell separation and probabilistic modeling, 
Forensic Sci. Int. Genet. 36 (2018) 205-212. https://doi.org/10.1016/j.fsigen.2018.07.004.

649

650

651

652

653

654

655

656

657

658

659

660

661

662

663

664

665

666

667

668

669

670

[20] T.J. Verdon, R.J. Mitchell, W. Chen, K. Xiao, R.A.H. van Oorschot, FACS separation of non-compromised forensically relevant biological mixtures, Forensic Sci. Int. Genet. 14 (2015) 194-200. https://doi.org/10.1016/j.fsigen.2014.10.019.

[21] V.R. Williamson, T.M. Laris, R. Romano, M.A. Marciano, Enhanced DNA mixture deconvolution of sexual offense samples using the DEPArray ${ }^{\mathrm{TM}}$ system, Forensic Sci. Int. Genet. 34 (2018) 265-276. https://doi.org/10.1016/j.fsigen.2018.03.001.

[22] K. Farash, E.K. Hanson, J. Ballantyne, Enhanced Genetic Analysis of Single Human Bioparticles Recovered by Simplified Micromanipulation from Forensic "Touch DNA" Evidence, J. Vis. Exp. (2015). https://doi.org/10.3791/52612.

[23] E.K. Hanson, J. Ballantyne, "Getting Blood from a Stone": Ultrasensitive Forensic DNA Profiling of Microscopic Bio-Particles Recovered from “Touch DNA” Evidence, in: Nucleic Acid Detect., 2013: pp. 3-17. https://doi.org/10.1007/978-1-62703-535-4_1.

[24] C.M. Grgicak, Analytical Thresholds: Determination of Minimum Distinguishable Signals, ISHI 2010 Mix. Work. (2010). https://strbase.nist.gov/mixture/3 - Analytical Threshold.pdf (accessed May 21, 2021).

[25] S. Noël, J. Noël, D. Granger, J.-F. Lefebvre, D. Séguin, STRmix ${ }^{\text {TM }}$ put to the test: 300000 non-contributor profiles compared to four-contributor DNA mixtures and the impact of replicates, Forensic Sci. Int. Genet. 41 (2019) 24-31. https://doi.org/10.1016/j.fsigen.2019.03.017.

[26] Institute of Environmental Science and Research Limited, STRmix v2.8 Implementation and Validation Guide, 2020.

[27] Laboratory for Forensic Technology Development and Integration, ProvedIt Database, 
(n.d.). https://lftdi.camden.rutgers.edu/provedit/files/ (accessed June 8, 2021).

672 [28] L.E. Alfonse, A.D. Garrett, D.S. Lun, K.R. Duffy, C.M. Grgicak, A large-scale dataset of single and mixed-source short tandem repeat profiles to inform human identification strategies: PROVEDIt, Forensic Sci. Int. Genet. 32 (2018) 62-70.

675 https://doi.org/10.1016/j.fsigen.2017.10.006.

[29] European Network of Forensic Science Institutes, ENFSI guidelines for evaluation and reporting in forensic science, 2015. https://enfsi.eu/wpcontent/uploads/2016/09/m1_guideline.pdf.

679

[30] Scientific Working Group on DNA Analysis Methods, Recommendations of the SWGDAM Ad Hoc Working Group on genotyping results reported as likelihood ratios, (2018). https://1ecb9588-ea6f-4feb-971a73265dbf079c.filesusr.com/ugd/4344b0_dd5221694d1448588dcd0937738c9e46.pdf.

[31] C.D. Steele, D.J. Balding, Statistical Evaluation of Forensic DNA Profile Evidence, Annu. 684 Rev. Stat. Its Appl. 1 (2014) 361-384. https://doi.org/10.1146/annurev-statistics-022513-

[32] C.C.G. Benschop, C.P. van der Beek, H.C. Meiland, A.G.M. van Gorp, A.A. Westen, T. Sijen, Low template STR typing: Effect of replicate number and consensus method on genotyping reliability and DNA database search results, Forensic Sci. Int. Genet. 5 (2011) 316-328. https://doi.org/10.1016/j.fsigen.2010.06.006.

$\varnothing$. Bleka, The theory behind EuroForMix and its functionalities, (n.d.). http://euroformix.com/sites/default/files/EuroForMix_Theory_v3.0.pdf (accessed June 8, 2021).

[34] S. Riman, H. Iyer, P.M. Vallone, Examining Discrimination Performance and Likelihood 
703

704

705

706

707

708

709

710

711

712

713

714

715

716

Ratio Values for Two Different Likelihood Ratio Systems Using the Provedit Dataset, (2021). https://doi.org/10.1101/2021.05.26.445891.

[35] T. Caragine, R. Mikulasovich, J. Tamariz, E. Bajda, J. Sebestyen, H. Baum, M. Prinz, Validation of Testing and Interpretation Protocols for Low Template DNA Samples Using AmpFeSTR® Identifiler®, Croat. Med. J. 50 (2009) 250-267. https://doi.org/10.3325/cmj.2009.50.250.

[36] J. Burrill, B. Daniel, N. Frascione, A review of trace "Touch DNA" deposits: Variability factors and an exploration of cellular composition, Forensic Sci. Int. Genet. 39 (2019) 818. https://doi.org/10.1016/j.fsigen.2018.11.019.

[37] P. Gill, Ø. Bleka, O. Hansson, C. Benschop, H. Haned, Forensic Practitioner's Guide to the Interpretation of Complex DNA Profiles, 2020.

[38] Scientific Working Group on DNA Analysis Methods, Guidelines for STR Enhanced Detection Methods, 2014. http://media.wix.com/ugd/4344b0_29feed748e3742a5a7112467cccec8dd.pdf. 


\section{Tables}

718 Table 1. Percentage of standard bulk mixtures in which all donors reach the specified

719 threshold

\begin{tabular}{|lcc|}
\hline Mixture Type $(\mathrm{n}=50)$ & $\log (\mathrm{LR})>0$ & $\log (\mathrm{LR}) \geq 6$ \\
\hline 2-person & $94 \%$ & $86 \%$ \\
\hline 3-person & $98 \%$ & $74 \%$ \\
\hline 4-person & $88 \%$ & $32 \%$ \\
\hline
\end{tabular}

Table 2. Percentage of Allele Drop-out per Locus

\begin{tabular}{|ccc|}
\hline \multicolumn{3}{|c|}{ Allele Drop-out } \\
\hline Locus & 1 Cells & 2 Cells \\
\hline D3S1358 & $52 \%$ & $25 \%$ \\
\hline vWA & $59 \%$ & $39 \%$ \\
\hline D16S539 & $68 \%$ & $44 \%$ \\
\hline CSF1PO & $66 \%$ & $45 \%$ \\
\hline TPOX & $74 \%$ & $54 \%$ \\
\hline AMEL & $36 \%$ & $24 \%$ \\
\hline D8S1179 & $50 \%$ & $31 \%$ \\
\hline D21S11 & $67 \%$ & $38 \%$ \\
\hline D18S51 & $61 \%$ & $39 \%$ \\
\hline D2S441 & $36 \%$ & $18 \%$ \\
\hline D19S433 & $39 \%$ & $25 \%$ \\
\hline TH01 & $57 \%$ & $34 \%$ \\
\hline FGA & $55 \%$ & $35 \%$ \\
\hline D22S1045 & $40 \%$ & $20 \%$ \\
\hline D5S818 & $48 \%$ & $35 \%$ \\
\hline D13S317 & $66 \%$ & $41 \%$ \\
\hline D7S820 & $67 \%$ & $46 \%$ \\
\hline SE33 & $75 \%$ & $56 \%$ \\
\hline D10S1248 & $43 \%$ & $25 \%$ \\
\hline D1S1656 & $52 \%$ & $36 \%$ \\
\hline D12S391 & $66 \%$ & $51 \%$ \\
\hline D2S1338 & $75 \%$ & $51 \%$ \\
\hline Total & $58 \%$ & $38 \%$ \\
\hline Avg. Alleles & 18 & 26 \\
\hline & & \\
\hline
\end{tabular}


727 Table 3. Percentage of subsamples and replicates analyzed with STRmix ${ }^{\text {TM }}$ that reach the 728 specified threshold

\begin{tabular}{|lcccc|}
\hline Sample Type & $\begin{array}{c}\text { Subsample } \\
\log (\mathrm{LR})>0\end{array}$ & $\begin{array}{c}\text { Replicate } \\
\end{array}$ & $\begin{array}{c}\text { Subsample } \\
\log (\mathrm{LR}) \geq 6\end{array}$ & $\begin{array}{c}\text { Replicate } \\
\text { SS 1 cell }(\mathrm{s}=100)\end{array}$ \\
\hline SS 2 cell $(\mathrm{s}=81)$ & $88 \%$ & $100 \%$ & $26 \%$ & $94 \%$ \\
\hline Mix 2 cell $(\mathrm{s}=110)$ & $60 \%$ & $100 \%$ & $60 \%$ & $93 \%$ \\
\hline
\end{tabular}


Deconvolution of Complex Mixtures by

Direct Single Cell Subsampling
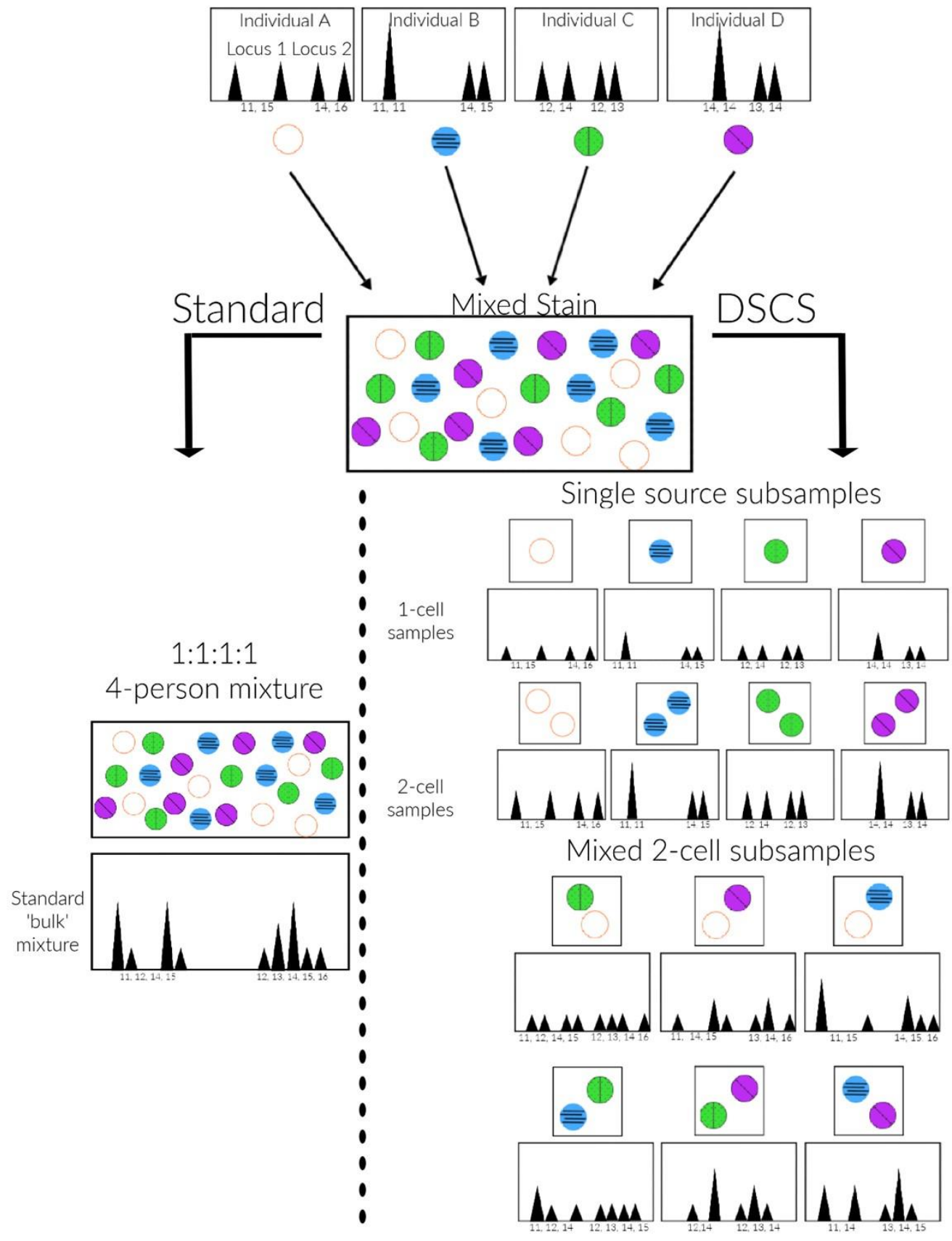


\section{PG Analysis Decision Tree}

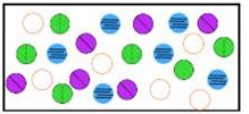

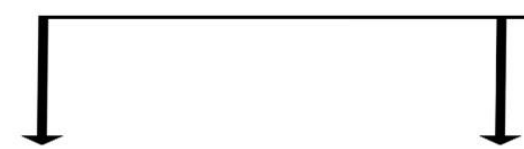

Standard extraction

from stain
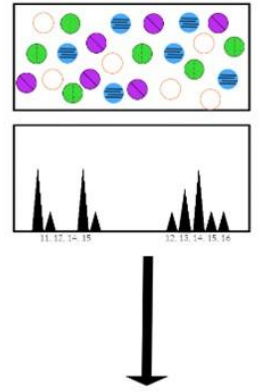

Traditional

PG analysis

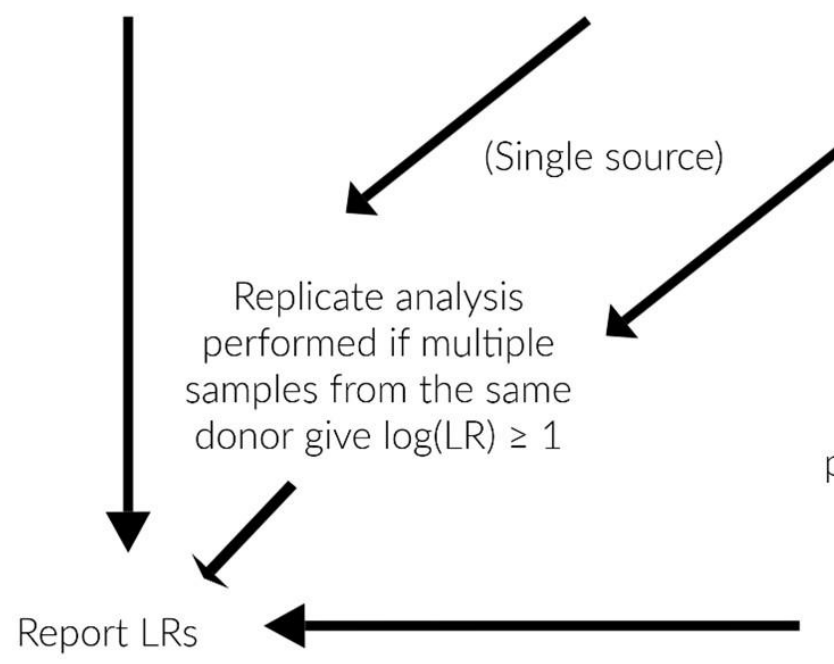

Collection of single cells

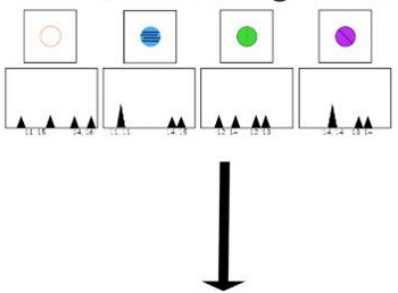

PG analysis validated for reduced rxn.volume $\&$ few cells

(1 contributor)

\section{Figure 2. Subsample types and associated probabilistic genotyping (PG) analysis method decision tree.}

Collection of 2-cells
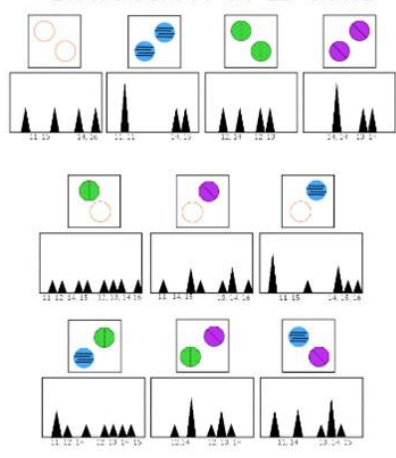

PG analysis validated for reduced rxn. volume \& few cells

(2 contributors)

Replicate analysis performed per donor on 'mini-mixtures' when multiple samples from the same donor gives

$$
\log (L R) \geq 1
$$




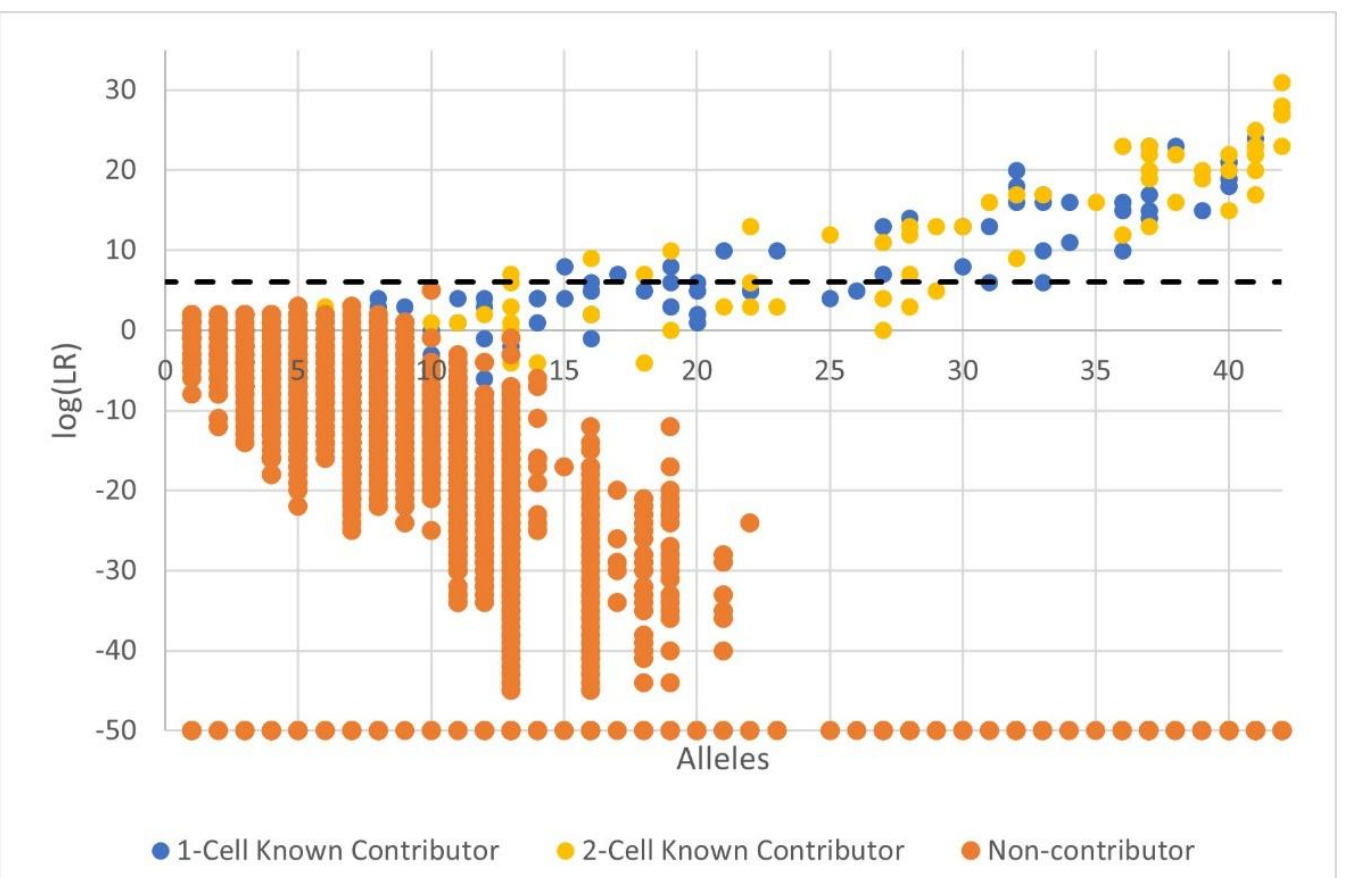

Figure 3. Sensitivity and specificity of DSCS (STRmix $\left.{ }^{\text {TM }}\right)$ analysis. Single source subsamples. $\mathrm{LR}=0$ plotted as -50 . Blue circles (1-cell known contributor), yellow circles (2cell known contributor), orange circles (non-contributor)

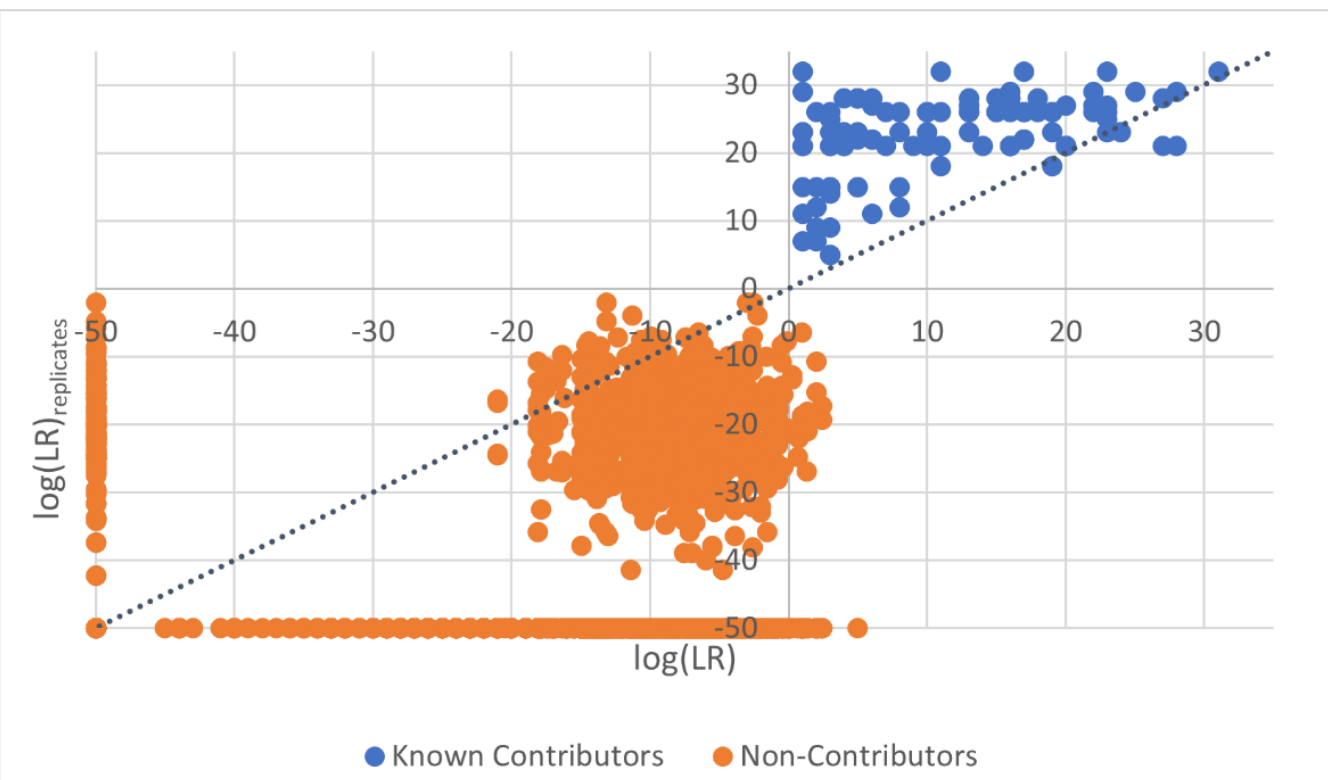

Figure 4. Specificity improvement of DSCS (STRmix $\left.{ }^{\mathrm{TM}}\right)$ with replicate analysis.

772

Known contributors above the diagonal (i.e. $\mathrm{y}=\mathrm{x}$ ) represent improved LR recovery from replicate

773 analysis. Single Source Cell Replicates. LR $=0$ plotted as -50 . Blue circles (known contributors);

774 orange circles (non-contributors) 


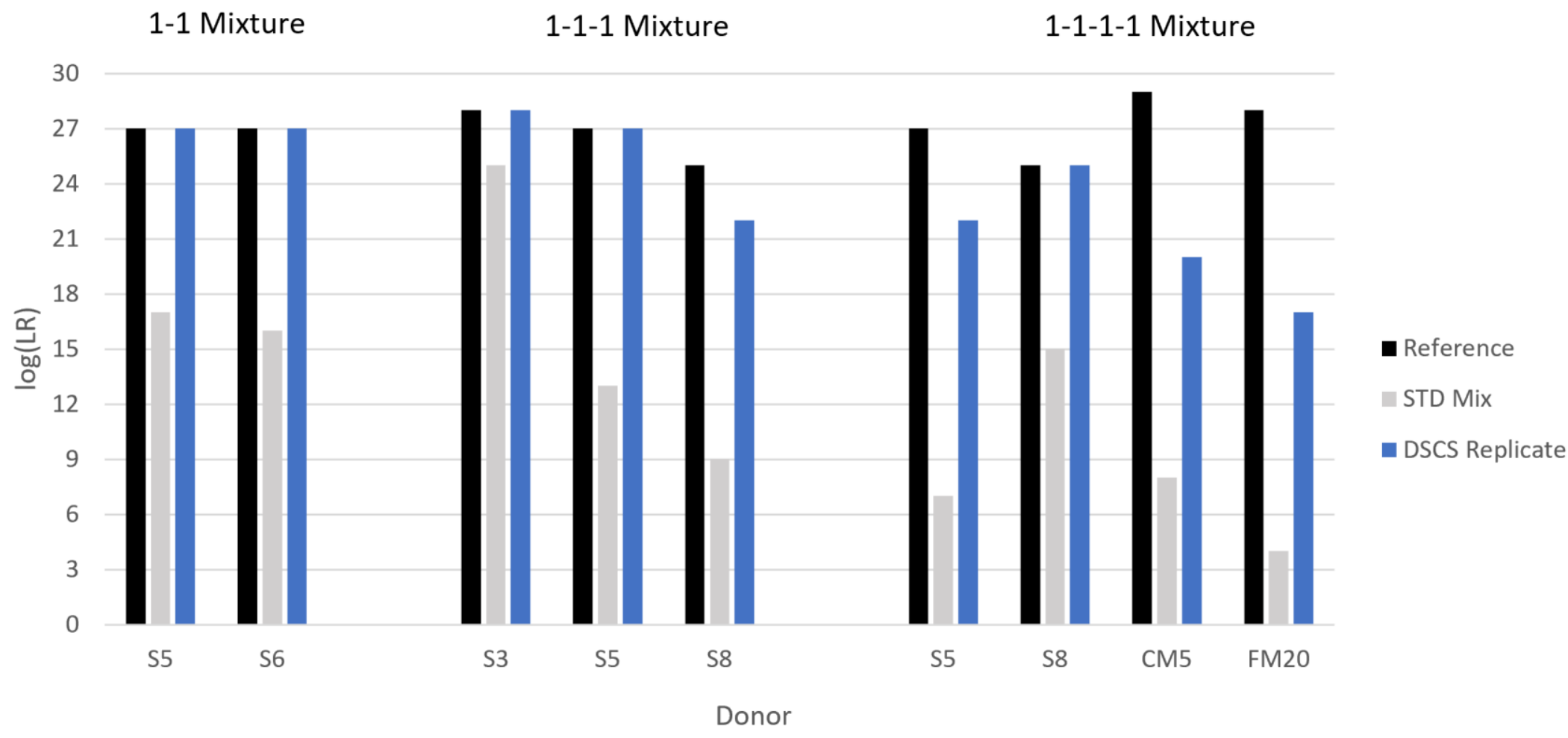

775

776

777

Figure 5. Increased contributor $\log (\mathrm{LR})$ recovery in 2-4 person mixtures by DSCS (STRmix $\left.{ }^{\mathrm{TM}}\right)$ compared to standard PG mixture analysis. 


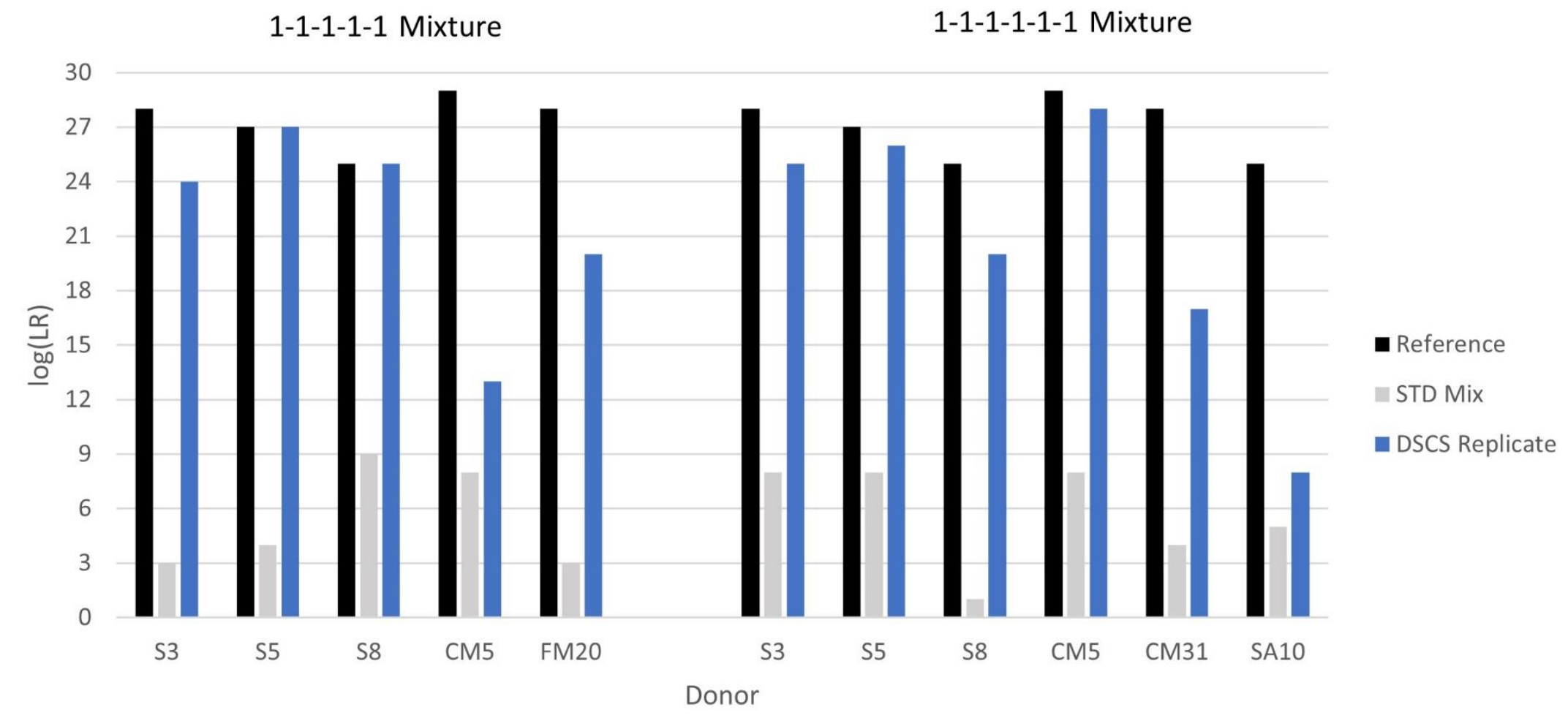

Figure 6. Increased contributor $\log (\mathrm{LR})$ recovery in 5-6 person mixtures by DSCS (STRmix $\left.{ }^{\mathrm{TM}}\right)$ compared to standard PG mixture analysis. 